\title{
Primary thyroid gland tuberculosis: a conundrum in a child
}

\begin{abstract}
Extrapulmonary tuberculosis (EPTB) is a great mimicker of innumerable diseases and it is often overlooked causing diagnostic dilemmas and therapeutic delay. Primary tuberculosis (TB) of the thyroid gland is a very rare form of EPTB with only a handful of cases documented in the world literature. Here, in this report, an unsuspected case of primary thyroid gland TB in a 7-year-old female is described which was misdiagnosed as a case of multinodular goitre.
\end{abstract}

Keywords: extrapulmonary tuberculosis, children, thyroid gland, cytology, multinodular goitre
Volume 8 Issue 3 - 2020

\author{
Sonam Sharma,' Rachit Sharma ${ }^{2}$ \\ 'Department of Pathology, Kalpana Chawla Government \\ Medical College, Karnal, Haryana, India \\ ${ }^{2}$ GRID Council, Delhi-NCR, India
}

Correspondence: Sonam Sharma, Department of Pathology, Kalpana Chawla Government Medical College, Karnal, Haryana, India,Tel 9999841393, Email drsonamsharma@gmail.com

Received: August 02, 2020 | Published: September 25, 2020
Abbreviations: TB, tuberculosis; EPTB, extrapulmonary TB; ESR, erythrocyte sedimentation rate; USG, ultrasonography; FNAC, fine needle aspiration cytology; AFB, acid-fast bacilli; MGG, maygrunwald giemsa; ATT, antitubercular treatment

\section{Introduction}

Tuberculosis (TB) is a multifaceted disease which mostly manifests as pulmonary $\mathrm{TB}$ and less frequently as extrapulmonary $\mathrm{TB}$ (EPTB). However, due to the escalation in the incidence of human immunodeficiency virus (HIV) infection and the emergence of drugresistant TB, the focus has now shifted towards the EPTB burden. EPTB can occur in isolation or along with a pulmonary focus, constituting $15 \%-20 \%$ of all the active cases. It occurs more commonly in immuno suppressed individuals and young children. Cervical tubercular lymphadenitis is the most common form of EPTB; nevertheless any body system can be affected. ${ }^{1-5}$ Thyroid gland TB, whether primary or secondary, is an extremely rare disease with a reported prevalence rate varying from $0.1 \%$ to $1 \%$, even in countries which have been declared as TB endemic zones. ${ }^{6}$ This is owing to the bactericidal property of colloid, high vascularity, iodine excess, well-developed capsule, enhanced activity of phagocytes in hyperthyroidism and possible antitubercular roles of thyroid hormone. ${ }^{7}$ TB is usually misdiagnosed at such atypical locations as the signs and symptoms are non-specific as well as it can masquerade several other clinical conditions resulting in diagnostic conundrum and unnecessary aggressive therapeutic interventions. One such experience of an extremely rare unsuspected case of primary thyroid gland TB in a 7-year-old female is reported herewith so as to create awareness about this enigmatic entity.

\section{Case report}

A 7-year-old female presented with a midline neck swelling since last 2 months associated with fever and fatigue. There was no history of anorexia or weight loss, dysphagia, respiratory or genitourinary symptoms, nervousness, tachycardia and palpitation. Her past, medical as well as family history was non-contributory. On physical examination, she had a body height of $128 \mathrm{~cm}$ and a body weight of $30 \mathrm{Kg}$ and a basal metabolic rate of $18.3 \mathrm{~kg} / \mathrm{m}^{2}$. Her vitals were within the normal limits and no pallor, icterus or any lymphadenopathy was evident. On local examination, there was a multinodular swelling in the thyroid gland region which was more prominent on the right side than the left and measured $4 \mathrm{~cm} \times 3 \mathrm{~cm}$ in size. The swelling moved up with deglutition. It was soft to firm in consistency and non-tender. The overlying skin was unremarkable Figure 1. No abnormality was detected on her systemic examination. The laboratory investigations revealed a haemoglobin level of $13.4 \mathrm{~g} / \mathrm{dl}$, total leukocyte count of $5300 / \mathrm{mm}^{3}$ with differential being of $52 \%$ lymphocytes and a platelet count of $510,000 / \mu 1$. The erythrocyte sedimentation rate (ESR) was 48 $\mathrm{mm} /$ first hour and the urine as well as blood cultures were negative. Renal as well as liver function tests were normal. T3, T4 and thyroid stimulating hormone were within the normal limits. Serum tests for antibodies to anti-thyroid peroxidase, human immunodeficiency virus, hepatitis B surface antigen and syphilis were negative. X-ray chest was normal. Ultrasonography (USG) of the thyroid gland region showed a diffusely enlarged thyroid gland with altered echotexture. Multiple ill-defined and few well-defined nodules were seen in both lobes. Largest nodule in right lobe measured $15 \mathrm{~mm} \times 11 \mathrm{~mm}$ and that in the left lobe measured $10 \mathrm{~mm}$ x $6 \mathrm{~mm}$. No cervical lymphadenopathy was seen. Based on these clinical, laboratory and radiological findings, a provisional diagnosis of multinodular goitre was made. To arrive at a conclusive diagnosis, Fine Needle Aspiration Cytology (FNAC) was performed. Two passes at two different sites of the lesion were done. Thick cheesy material was aspirated in both the attempts. This material was pushed onto the clean glass slides and the smears were drawn. The smears prepared, were stained with May-Grunwald Giemsa (MGG) stain followed by microscopic examination. FNAC smears revealed numerous epithelioid cell granulomas with peripheral lymphocytic cuffing, langhans giant cells and caseating necrosis. Scattered clusters of benign thyroid follicular epithelial cells were also seen in the background Figure 2. Special stain for tubercle bacilli (Ziehl-Neelsen stain) showed few acid-fast bacilli (AFB) Figure 2-Inset. Based on these cytological features, a final diagnosis of thyroid gland TB was established. She was investigated further to look for possible sites harbouring the tubercular infection. However, no AFB was found in her sputum as well as urine samples. Mantoux test resulted in $10 \mathrm{~mm}$ induration. On abdominal USG, no lymph node enlargement was seen. The patient was put on standard short course antitubercular treatment (ATT), to which she responded well as there was a gradual regression in the thyroid gland size in subsequent follow-ups. 


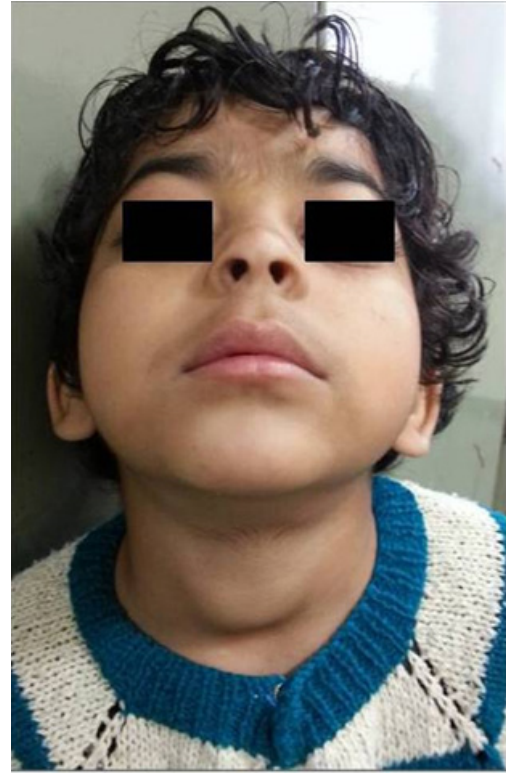

Figure I Midline neck swelling on clinical inspection.

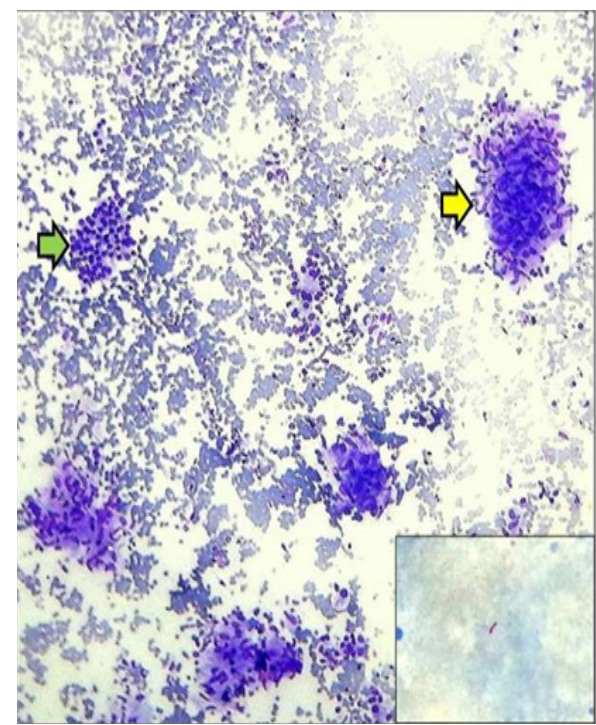

Figure 2 Epithelioid cell granuloma (yellow arrow) and benign thyroid follicular epithelial cells (green arrow) (MGG, xl00). [Inset: Ziehl-Neelsen stain showing presence of AFB].

\section{Discussion}

Thyroid gland TB is rare form of EPTB which was first documented in the world literature in the year 1862 by Lebert in a military TB patient. ${ }^{8}$ Since then, there have been many isolated case reports and few case series pertaining to it in the literature. ${ }^{7,9}$ The pathogenesis behind the dissemination of tubercle bacilli to the thyroid gland is controversial. It usually occurs in association with tuberculous infection of the other tissues or organs. For the organisms to reach the thyroid gland, the spread is either through the hematogenous or lymphatic route or there can be a direct extension from an active laryngeal or nodal focus. ${ }^{10} \mathrm{~TB}$ primarily affecting the thyroid gland is much more uncommon and, predictably, more difficult to diagnose. ${ }^{11}$ Nevertheless, various pathological varieties of thyroid gland lesions in tuberculous inflammation have been described, which includes the miliary spread to the thyroid gland as a part of generalized dissemination, enlargement of the thyroid gland due to caseating granulomas developing in adenomatous type of thyroid, cold abscess formation, sometimes associated with multiple sinuses, chronic fibrosing TB and acute abscess formation. ${ }^{6,12}$ On further exploring the existing literature on thyroid gland $\mathrm{TB}$, certain clinicopathological features of this condition draw special attention, which in turn can help in its correct diagnosis and treatment.

Clinically, all the age groups can be involved. The reported median age for men is $40 \pm 16$ years while that for women is $44 \pm 17$ years. However, the children constitute very small proportion of these cases, with the youngest documented case being that of a 5-month old female infant. ${ }^{7,8,13}$ These patients can be asymptomatic or may have an acute or subacute presentations, the duration of which varies from two weeks to one year and has no relationship with the patient's age or sex. The most common symptoms are fatigue, fever, night sweats and weight loss. Nevertheless, pressure symptoms, like dysphagia, dyspnea, dysphonia can occur. This disease can also manifest as "a clinical syndrome of hyperthyroidism" which generally occurs at the beginning of glandular involvement due to its destruction while hypothyroidism is caused by extensive glandular destruction by caseous necrosis. ${ }^{10,12}$ However, these wide varieties of manifestations are quite non-specific and can mimic other clinical conditions, therefore, a high index of suspicion is required for early diagnosis of this rare entity. For clinching its definite diagnosis, a collaboration of clinical history with all the radiological, cytological, histopathological investigations is required. Clinically, a gradual enlargement of thyroid gland associated with a past history of TB concomitant with cervical lymphadenopathy or any other site of tuberculous involvement might lead to a correct clinical diagnosis. The presence or absence of local pain is also an important clinical finding, which helps in the differential diagnosis as if the pain predominates, the diagnosis lies between an infectious form of thyroiditis and subacute granulomatous thyroiditis. In the event where pain is absent, thyroid gland TB might be falsely diagnosed as thyroid malignancy, nevertheless, the two conditions may even co-exist. ${ }^{14}$

However, once thyroid gland TB is clinically suspected, among all the laboratory tests, ESR, the tuberculin skin test, urine as well as blood cultures can provide a vital clue for its diagnosis. The thyroid function tests are usually normal as the thyroid function is preserved in majority of the cases, however, thyroid hormones abnormalities can rarely occur in such an unusual setting. ${ }^{6}$ Imaging techniques such as chest X-ray, USG, computed tomography, and magnetic resonance imaging, although not specific, are extremely helpful in its assessment. ${ }^{15}$ The role of FNAC in differentiating all the thyroid lesions is quite well-established. ${ }^{16-22}$ Likewise, cytology is also pivotal in diagnosing thyroid gland TB and preventing unnecessary surgery. ${ }^{7,23}$ Nevertheless, the reported diagnostic rate of the thyroid gland TB by FNAC is $0.6 \%-1.15 \%$ among the thyroid gland lesions in endemic regions..$^{10}$ Researchers have also suggested that USG guided FNAC should be usually practised in such a case of a highly vascular organ like thyroid gland so as to avoid hemodilution and to get better yield of diagnostic material..$^{15,24}$ The characteristic feature of thyroid gland TB on cytology is the presence of caseating epithelioid granulomas accompanied by lymphocytic infiltration and multinucleated Langhan's type giant cells. Although these features can be seen in several other thyroid gland pathologies like de Quervain's thyroiditis etc., nevertheless, caseous necrosis even if focally present should raise a suspicion of TB. ${ }^{6,25}$ The simultaneous demonstration of AFB by Ziehl-Neelsen staining also makes its diagnosis almost certain.

Furthermore, a mycobacterial culture and enzyme amplification (polymerase chain reaction) from the aspirated material can be useful for confirming its diagnosis. ${ }^{15}$ In rare instances where FNAC 
fails in diagnosing thyroid gland TB, the diagnosis is usually made only after histopathological examination of the surgical specimen. ${ }^{6}$ However, it has also been mentioned to be an incidental postoperative histopathological surprise after the patients have undergone thyroidectomy for some other cause. ${ }^{26}$ Seed L in the year 1939 also proposed the criteria for diagnosing thyroid gland $\mathrm{TB}$, which included: 1) demonstration of AFB within the thyroid gland, 2) a necrotic or abscessed gland, 3) demonstration of tuberculous focus, outside the thyroid gland. ${ }^{27}$ Histological and bacteriological confirmation is adequate to make its diagnosis but the fulfilment of third criterion is not essential. However, nowadays it has been observed that AFB are not always found, therefore, multiple coalesced and caseated epithelioid cell granulomas along with giant cells are considered to be diagnostic of tuberculous affection of the thyroid gland, which further helps in differentiating it from other granulomatous inflammatory conditions of the thyroid gland, like granulomatous thyroiditis, palpation thyroiditis, fungal infection, sarcoidosis, granulomatous vasculitis, and foreign body reaction. ${ }^{6,23}$ Therapeutically, the treatment of this rare entity initially consisted of ATT combined with surgical removal of the affected parts of the thyroid gland or surgical drainage. ${ }^{28}$ However, it has been now recognized that a complete resolution of this disease is usually achieved by an appropriate ATT alone. ${ }^{29}$ But in cases with large abscess, surgical drainage or resection followed by ATT is still considered as sufficient, and further surgery is rarely required. ${ }^{30}$ The present case presented with a painless multinodular thyroid gland swelling and a few constitutional symptoms. Her ESR was raised and she was euthyroid; however, she was misdiagnosed as a case of multinodular goitre, as the radiological investigations were inconclusive of TB. It was only on cytological evaluation that a diagnosis of thyroid gland TB could be established and an early ATT was instituted, to which she responded well.

\section{Conclusion}

Thyroid gland TB is an exceptionally extraordinary entity which should always be kept in mind while dealing with thyroid gland swellings, especially in people residing in TB endemic areas irrespective of their age. Further, it is essential that a detailed clinical history and meticulous workup of such patients should be done for timely intervention, suitable treatment, and contact tracing.

\section{Acknowledgments}

None.

\section{Conflicts of interest}

The authors declare that they have no conflicts of interest.

\section{Funding}

None.

\section{References}

1. Sharma S, Sharma S. Concomitant madura foot and tuberculosis in a child: a diagnostic dilemma! J Foot Ankle Surg (Asia-Pacific). 2014;1(2):69-71.

2. Bhasin TS, Mannan R, Sharma S, et al. Extra-pulmonary tuberculosis presenting as established non union tibia shaft fracture. Nat J Lab Med. 2016;5(4):PC01-PC03.

3. Sharma S, Dutta S, Yadav AK, et al. A rare case of cervical tuberculosis masquerading as carcinoma cervix. Ann Woman Child Health. 2016;2(2):C20-C23.
4. Sharma S. Spermatic cord tuberculosis: The great masquerader. Int $J$ Mycobacteriol. 2019;8(2):196-198.

5. Sharma S, Kalyan S. Primary bilateral maxillary sinus tuberculosis: a rare and an underdiagnosed entity. Int J Fam Commun Med. 2020;4(2):48-50.

6. Majid U, Islam N. Thyroid tuberculosis: a case series and a review of the literature. J Thyroid Res. 2011:359864.

7. Bodh A, Sharma N, Negi L, et al. Thyroid tuberculosis in a child: a rare entity. J Lab Physicians. 2014;6(1):40-42.

8. Razmpa E, Sharifian H, Sadeghi-Hasanabadi M, et al. Clinical and paraclinical aspects of thyroid tuberculosis. Acta Med Iran. 2007;45(5):389-394.

9. Jiang YD, Gao JQ, Chen S, et al. Thyroid tuberculosis: two cases report and review of the literature. Int J Clin Exp Med.2016;9(11):22477-22485.

10. Kapan M, Toksöz M, Bakır ŞD, et al. Primary thyroid tuberculosis. Eur J Gen Med. 2011;8(4):357-360.

11. Zendah I, Daghfous H, Mrad SB, et al. Primary tuberculosis of the thyroid gland. Hormones (Athens). 2008;7(4):330-333.

12. Lindsay LM, Mead CI. Tuberculosis of the thyroid gland with report of a case in a child aged three. Can Med Assoc J. 1934;30(4):373-377.

13. Sood AK, Chauhan L, Aggarwal N. Tuberculosis of the thyroid gland in an adolescent girl with disseminated tuberculosis. Pediatr Infect Dis J. 2015;34(5):550-551.

14. Suri VS, Sakhuja P, Malhotra V, et al. Co-existent tuberculosis and papillary carcinoma thyroid. Trop Doct. 2002;32(2):118.

15. Majumder A, Sen D. Uncommon affliction of a common disease-primary tuberculosis of thyroid gland. Indian J Pathol Microbiol. 2020;63(1):8385 .

16. Bhasin T, Mannan R, Manjari M, et al. Reproducibility of 'the Bethesda system for reporting thyroid cytopathology': a multicenter study with review of the literature. J Clin Diagn Res. 2013;7(6):1051-1054.

17. Sharma S, Mandal D, Yadav AK, et al. Primary thyroid lymphoma: a comprehensive summary of two cases. Int J Sci Rep. 2015;1(3):177-180.

18. Mannan R, Piplani S, Sharma S, et al. Cytological correlation of spectrum of head and neck lesions with epidemiological and diagnostic parameters. Indian J Pathol Oncol. 2017;4(1):92-97.

19. Kaur J, Sharma S, Bhasin TS, et al. A rare incidental case of an occult breast carcinoma micrometastasis in papillary thyroid carcinoma: A view within a view. Thyroid Res Pract. 2018;15(3):142-146.

20. Singh P, Mittal MK, Sharma S. Thyrolipoma: a rare thyroid gland entity. Nepalese Journal of Radiology. 2019;9(13):24-29.

21. Sharma S, Singh M, Bhuyan G, et al. Extragonadal dysgerminoma presenting as neck metastasis and masquerading as a thyroid swelling. Clin Cancer Investig J. 2016;5(1):43-45.

22. Sharma S, Ahluwalia C, Singh M, et al. Diagnostic utility and efficacy of conventional versus surepath ${ }^{\circledR}$ liquid-based cytology in head and neck pathology: a study in an Indian tertiary care hospital. Iran J Pathol. 2018;13(2):188-195.

23. Simkus A. Thyroid tuberculosis. Medicina (Kaunas). 2004;40(3):201204.

24. Mondal A, Patra DK. Efficacy of fine needle aspiration cytology in the diagnosis of tuberculosis of the thyroid gland: a study of 18 cases. $J$ Laryngol Otol. 1995;109(1):36-38.

25. Terzidis K, Tourli P, Kiapekou E, et al. Thyroid tuberculosis. Hormones (Athens). 2007;6(1):75-79. 
26. Sharma AB, Kumar DL, Sharma HD, et al. Primary tuberculosis of the thyroid gland-a rarity. JIACM. 2006;7(4):363-364.

27. Seed L. In goldberg's clinical tuberculosis. 2nd Edition. Philadelphia: FA Davis Co. 1939.

28. Kukreja HK, Sharma ML. Primary tuberculosis of thyroid gland. Ind $J$ Surg. 1982;44(3):190-192.
29. El Malki HO, El Absi M, Mohsine R, et al. Tuberculosis of the thyroid. diagnosis and treatment. Ann Chir. 2002;127(5):385-387.

30. uz-Zaman M, Hussain R, Mirza MK, et al. Isolated tuberculous thyroiditis as solitary thyroid nodule. J Coll Physicians Surg Pak. 2008;18(2):121122. 